\title{
New and future interactivity in ship project
}

\author{
Valerio Ruggiero ${ }^{1}$
}

Received: 18 October 2019 / Accepted: 21 October 2019 / Published online: 31 October 2019

○) Springer-Verlag France SAS, part of Springer Nature 2019

\section{Introduction}

Since the beginning a Ship project and design needed a deep interactivity among several elements: a Ship is not just a transportation tool, she is considered a feminine character, something alive, in almost all the marine cultures, that's why a ship is "SHE" and not "IT". In the Italian culture there is also a "gender difference" among the commercial ships and the navy vessels, the same word "nave" becomes female or male depending from the final use of the ship. This "soul" of the ship is probably due the particular behavior of the ship that has always had to operate in a scenario extremely varying from the calm inland waters to the severe storms in Oceans. Furthermore, as well known and demonstrated in Naval Architecture, a ship has to interact with the forces of Nature, and the response in terms of movements on the 6 degrees of freedom ( 3 translations and 3 rotations with an $\mathrm{XYZ}$ axis system) is strictly related. Also the main principle of the ship floatation, discovered by Archimede many centuries ago, is an example of interactivity between the geometrical shape of the ship and her environment.

But, furthermore, there is a fact that cannot be neglected: a ship must be operated by human beings and carry human beings, so she has to deal not only with the natural environment, but also with the specific needs of the human beings in terms of ergonomics, safety and so on.

Additionally, the ship has the singularity of operating on the separation layer between two different fluids, air and water, whose densities differ of about 800 times, and this aspect, with all the consequences in terms of mathematical models, leaded to the theory, still valid and used, of a prediction of resistance that with the separation of the different coefficients for frictional resistance and residual resistance, related to Reynolds number and Froude number, is a perfect

Valerio Ruggiero

vruggiero@unime.it

1 Department of Ingegneria, University of Messina, Messina, Italy example of interaction between theoretical and experimental approach.

But in the last decades ships presented extremely fast changes: if we consider the evolution of the sails and shapes of ships until 1492, the discovery of the Americas and the following transoceanic voyages, then the timeframe until the application of steam propulsion and, later on, the development of globalization after the 2nd world war, we can note that each "age" has being shortening from centuries to decades to years. We passed from the sail wood ships $20-30 \mathrm{~m}$ long, that lasted, with some differences, for decades, to modern steel ships, 300-400 m long, with shorter lives. In particular, in the last 20-30 years, passengers ships have been impressively increasing in terms in gross tonnage. In Fig. 1 this is evident comparing the sizes of a modern cruise ship and a transoceanic liner.

Briefly summarizing, it is clear that the degree of actual interactivity among the various steps of project and design is deeply changed, not only because of the different needs, but also because of the different tools that can be used for the different steps: CAD, CAM, CFD, FEM analysis made possible to design a component of the ship and immediately verify the impact of this component on the whole project.

The present paper's aim, as introduction to the papers contained in this issue, is to explore and describe the impact of these changes on the approach to the design, underlining the most recent topics and suggesting and exploring the new topics who became mandatory in the last years and suggesting which topics could be integrated in the future in terms of interactivity among the ship project and the "rest of the world needs".

\section{The main changes and consequences}

For decades the main challenges for the project were focused on the needs of the passengers/utilizers of the ship and the cost containing for the builder; in this way all the efforts were finalized to safety, to the time reduction for design and 


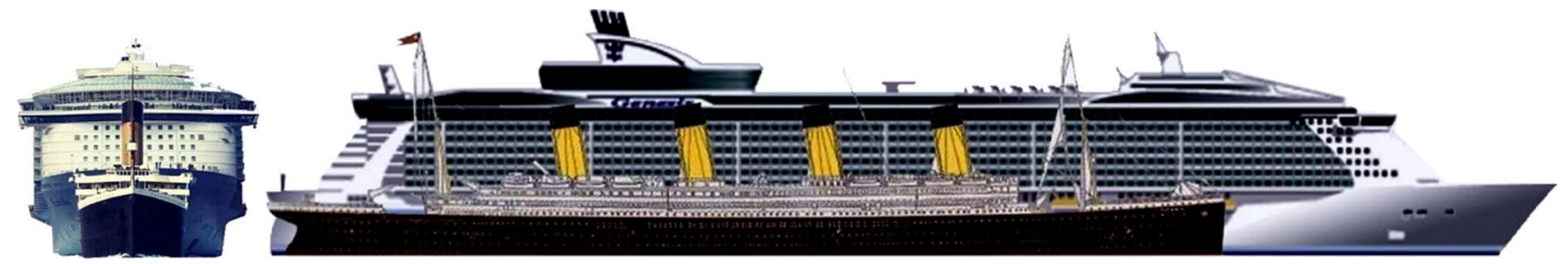

Fig. 1 Comparing sizes of a modern cruise ship and a transoceanic liner

Fig. 2 Ship design spiral example, Image available on web

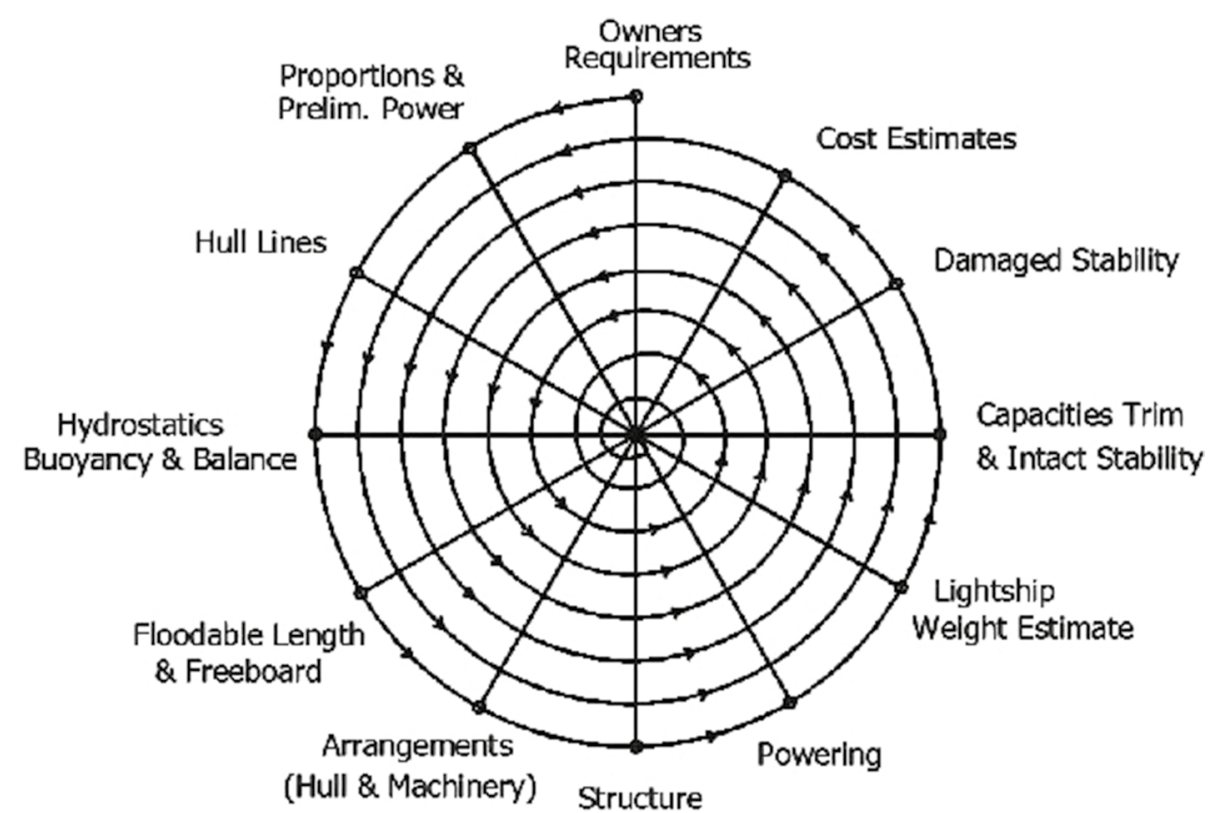

building and to the capability to maintain the ship in good operation during her life.

In this time it has been developed an approach called the "ship design spiral" [1] where the degree of interactivity in the design was basically among the various steps : the first definition of the main dimension was subject to a design of the general arrangement, consequentially to a verification of the Body plan, then to a preliminary weight estimation and, subsequently to a preliminary stability check, consequentially to a prevision of power installed, and then back to the Body plan adding at each step a new segment or station to be verified (Fig. 2).

Actually the showed items are changing continuously and there is a complete new field to explore: it is mandatory now to operate all the optimizations described technically, but the new priority, for every kind of passenger, commercial, oceanic, inland water ships and so on, is the "environment protection".

In the last 10 year the protection of the environment became the "main target" to aim at, during all the stages of project and construction: the IMO 2010 limits on Sulphur contents (Fig. 3) will aim at the reduction of $80 \%$ of pollution from Sulphur [2]; commercial ships now have to

\begin{tabular}{|l|c|c|}
\hline \multirow{2}{*}{ Date } & \multicolumn{2}{|c|}{ Sulfur Limit in Fuel $(\% \mathrm{~m} / \mathrm{m})$} \\
\cline { 2 - 2 } & SOx ECA & Global \\
\hline 2000 & $1.5 \%$ & $4.5 \%$ \\
\hline 2010.07 & $1.0 \%$ & \\
\hline 2012 & & $3.5 \%$ \\
\cline { 1 - 1 } 2015 & \multirow{2}{*}{$0.1 \%$} & \\
\hline 2020 & & $0.5 \%$ \\
\hline
\end{tabular}

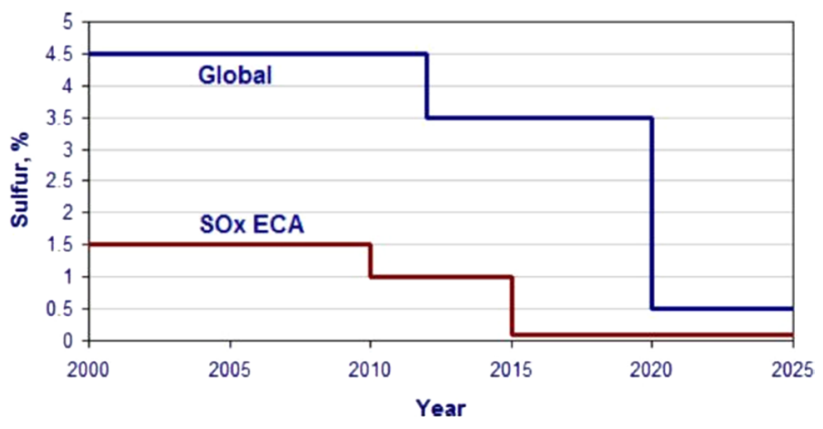

Fig. 3 IMO MARPOL Annex VI Limits

operate according to the limits and concepts of the EEDI Energy Efficiency Design Index and EEOI Energy Efficiency Operating Index [3]. 
The two index EEDI and EEOI, are indicators of the capability of the ship to optimize the energy consumption during her life, and they introduce a further step of interactivity during the life of the ship, declaring a "rule of interactivity" between the results during the Design (EEDI) and the results during the operational life (EEOI).

The line guide of the Indian Register of shipping [4], for example, in Fig. 4, shows a very easily understandable example of interactivity between design and realization to achieve good results in terms of International design index certificate:

This approach it is not only applicable to merchant ships, but it is involving every type of ship, also the so called "pleasure ships", the new IMO TIER Rules for engines being applied also to yachts [5] and small passenger vessels, operating also in internal waters.

The logic consequence of all these new requirements and limits is that the interactive process of the Design spiral needs to be seen from a different perspective and probably needs some changes to the approach, because the "stations" to verify are becoming too many and too much related.

\section{Interactive approach in the future}

All the above described elements, in the reality of facts, have been overcome by the technological progress: the shipyard and the owner are now capable to begin with a multidisciplinary approach to the problems of reducing the emissions using different technologies.

Most part of the new ships are already installing new propulsion system with natural gas or with extensive use of batteries in order to point at "almost zero emissions".

This approach it is a real, realized example of interaction: the new hybrid propulsion systems are taking great advantage from the discoveries as for the optimization of batteries, using the new generation of Li-Io batteries.

As for large ships new solutions are studied to reduce emissions during the moored time at pier, providing energy from stations ashore.

Propulsion engines are studied from beginning to operate with gas fuel.

Those new solutions significantly changed the previous approach: it is difficult now to apply the classic interactive approach of choosing a solution and then verify it: the interaction must be planned with the new future rules, and needs several solutions to be applied and verified simultaneously, otherwise the variables will effect in a significant way the final result.

Several modifications to the Design spiral have been studied, like for example the "Blackboard system" [6], using a matrix/blackboard process to cohordinate the various elements.

What become clear from the analysis of the previous described new criteria of design and Rules of environmental protection and the various methods adopted, it is the need to "look forward": not only apply a process of design-verify but also to preview wich changes and new requests will appear and integrate them in the design process.

The bottom line, and the main item to consider, no matter wich system will be used, it is now the need to foreseen the Environmental impact of the ship during her life, but this aspect opens to a new challenge for the designers, that will be a fundamental change in last years, and this can be considered the "innovative interaction" that must be examined: the long (short?) time future.

Infact, it is necessary consider what will happen to the ship and to her components at the end of the life: the images of "ship cemetery" in third world countries, with ship dismounted by people without respecting any rules for safety, human and environmental, will be no more acceptable, but a $360^{\circ}$ degree approach to the design will have necessarily to consider the problem of the "end life" of the ship, with the recycle, where possible, of all the elements used for the construction. But with the complication of the use of the new materials: steel, aluminium alloy, are metals and the know how to deal with their recycle it is well know, different approach could be considered the problem of recycling the composites used for smaller constructions, interiors.

For example, referring to what now is a major trend of several ships, the hybrid propulsion, designer will have to plan already that the new hybrid constructions with the installation on board of battery pack of several $\mathrm{MWh}$, will need soon, considering an estimated life cycle of battery packs of 5-10 years, an organized solution for the removing of them and the consequent recycle if possible.

All those problems and needs will lead to an higher level of interactivity among all the elements constituting the ships and among the ship and the environment, developing in an organic and armonic way those interactions will be certainly the main challenge for the future. 


\section{$\underline{\text { International Energy Efficiency Certificate }}$}

\section{Certification Process - Flow Chart}

$\underline{\text { Stage 1: Design Stage }}$

Request from shipyard for International Energy Efficiency

Certificate (as part of SRF submission)

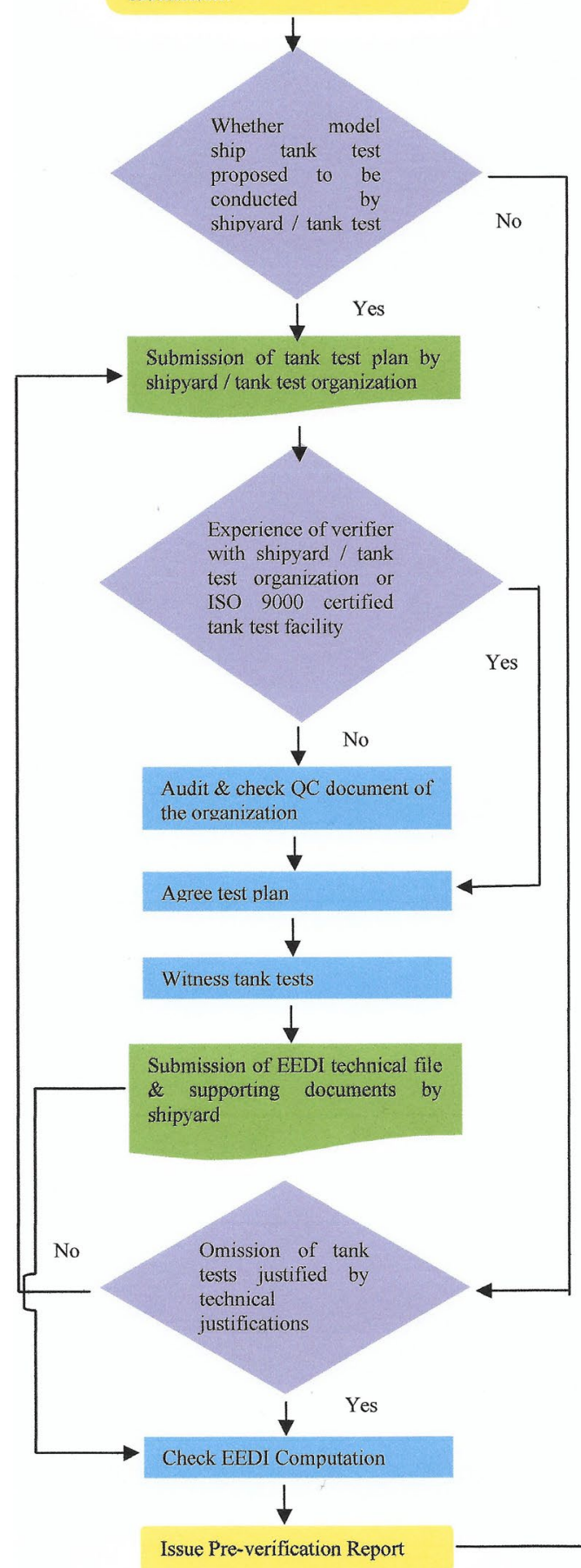

Stage 2: Sea trials Stage

Submission of documents prior to

Sea trial

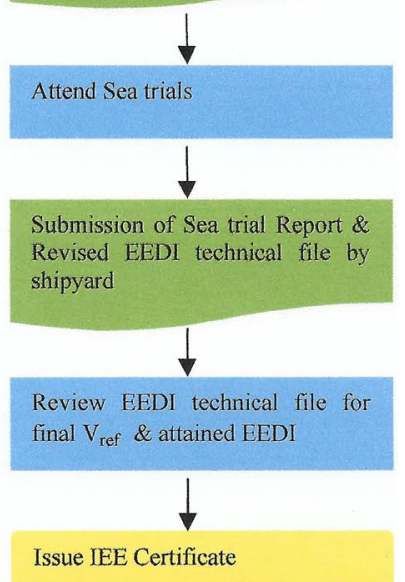

Fig. 4 Flow chart suggested by Indian Register to obtain EEI certificate 


\section{References}

1. Evans, J.H.: Basic design concepts. J. Am. Soc. Nav. Eng. (ASNE) 74, 671-678 (1959)

2. IMO-International Maritime Organization - MEPC.1/Cir.878 -9 (2018)

3. IMO-International Maritime Organization - MEPC 245(66) 2014 Guidelines for the calculation of attained EEDI for new ships (2014)

4. Ruggiero V.: The impact of latest rules in environmental pollution on the project of mega yachts. In: IV International Multidisciplinary Scientific Conference on Social Sciences and arts-SGEM
2017, book 5, volume I, Vienna 28-31 March 2017, Stef 92 Technology Ltd, Sofia (Bulgaria), ISBN 978-619-7105-964 (2017)

5. Implement Energy Efficiency Design Index.: Indian register of shipping (2018)

6. Vassalos, D., Oestvik, I., Konovessis, D.: Recent developments and application of a formalized design for safety methodology in an integrated system. SNAME Trans. 2000, 419-5445 (2000)

Publisher's Note Springer Nature remains neutral with regard to jurisdictional claims in published maps and institutional affiliations. 